\title{
Seasonality of 278 neuromyelitis optica relapses in a Brazilian cohort
}

\author{
Sazonalidade de 278 surtos em pacientes brasileiros com neuromielite óptica
}

Bichuetti DB has received speaking honoraria from Bayer Health Care, Merck Serono and Biogen Idec, serves in advisory board meetings by Merck Serono and Sanofi Genzyme and had travel expenses to scientific meetings paid by Bayer Health Care, Merck Serono and TEVA. Souza NA had travel expenses to scientific meetings paid by Bayer Health Care, Merck Serono and TEVA. Oliveira EML received compensations for participating in meetings sponsored by Bayer Health Care, Biogen Idec, Merck Serono, and Teva Pharmaceuticals and serves in advisory board meetings by Biogen Idec and Novartis. Fragomeni MO has nothing to disclose. This is not an industry-sponsored study.

Neuromyelitis optica (NMO) is and autoimmune disease with distinct, clinical, radiological and genetic profile when compared to multiple sclerosis $(\mathrm{MS})^{1}$ and, although much is known about its pathophysiology, the influence of environmental factors in patients with NMO is not well established as in $\mathrm{MS}^{2}$. It has been previously demonstrated that patients with MS present a higher birth prevalence for months in the spring and summer, as well as there is a higher prevalence of MS relapses in these same months ${ }^{3,4}$, but the monthly rate of relapses in patients with NMO has been described in only one study with distinct result from $\mathrm{MS}^{5}$ and has not been confirmed with further studies.

To evaluate if there is any yearly variation in the incidence of relapses in patients with NMO, we reviewed our historical series published in $2009^{6}$, as it contains detailed information of all relapses suffered by each patient and added information on 8 patients from a pediatric NMO cohort that is under review in our center. All patients received regular preventive therapies recommended at the time they were seen, which included, corticosteroids, azathioprine, cyclophosphamide, methotrexate, plasma exchange and IV immunoglobulin? This work is part of an continuous observational project at the Universidade Federal de São Paulo, has received local ethic committee approval and patients have given informed consent for retrospective and prospective data collection.

We retrieved 282 relapses from the 49 patients (41 with adult onset, $41 \% \mathrm{NMO}-\mathrm{IgG}+$; and 8 with pediatric onset,
75\% NMO-IgG+) with relapsing NMO, but analyzed only 278 relapses (98\%), as four of them did not have the month of occurrence available in their medical record. The adult onset information was retrieved from the historical cohort seen from 1994 to $2007^{6}$ and the pediatric onset patients were seen from 2002 to 2013. The statistical analysis was performed using Prism 5 (GraphPad Software Incorporated $\left.{ }^{\circledR}\right)$; relapses were pooled in two groups representing seasonality already observed in MS studies ${ }^{4}$ : spring/summer and fall/winter months. The Kolmogorov-Smirnov test was used to confirm a normal distribution of the sample and the unpaired t test performed for comparing both groups.

The seasonality for the 278 relapses analyzed was: $21 \%$ in spring (September - November), 24\% in summer (December - February), 27\% in fall (March - May) and 28\% in winter (June - August) (Figure). Although there seemed to be a higher incidence of relapses in the fall/winter months as opposed to spring/summer (55\% x 45\%), this difference did not reach statistical significance $(\mathrm{p}=0.11$, unpaired $\mathrm{t}$ test comparing relapses grouped in spring/summer and fall/winter months), disclosing no differences along the year. This lack of variation could be due to low sunlight exposure variation (if indeed there really is some seasonality influence on NMO relapses), as the city of São Paulo is located in a subtropical climate zone with low sunlight variation when compared to higher latitude cities (Latitude $-23^{\circ} 32$ ' $51^{\prime \prime} \mathrm{S}$, Longitude $-46^{\circ} 38^{\prime} 10^{\prime \prime} \mathrm{W}, 760$ meters above sea level), or simply signifies that sun exposure does not influence relapses in NMO as it does in MS, as also observed by Muto et al in Japan $^{5}$. A similar analysis with MS patients from Campinas, a city located less than $100 \mathrm{~km}$ from Sao Paulo disclosed higher relapse incidence in the summer/spring months, suggesting that climate variation in the southeast of Brazil can influence disease activity as elsewhere ${ }^{4}$, corroborating the idea that climate might not influence NMO relapses. Since this analysis comes from a historical cohort, information on vitamin D levels was not available and this should be prospectively evaluated together with other factors, such as the association to common infection and physical and psychological 
Relapses incidence by month

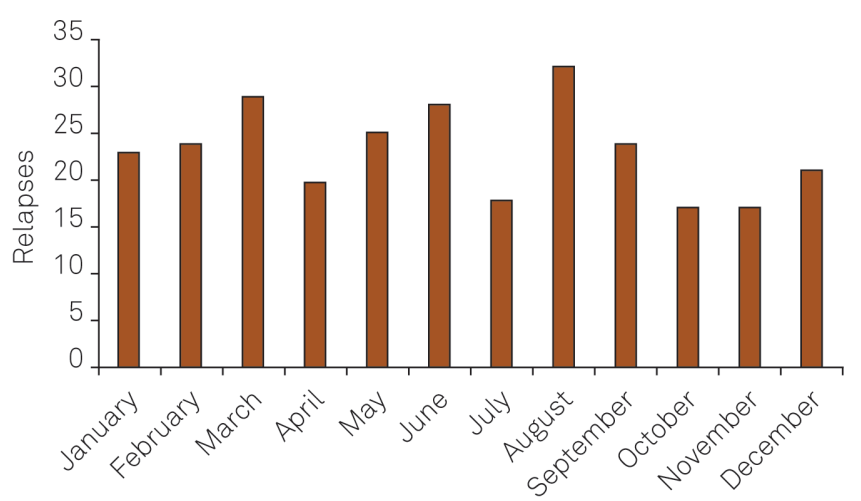

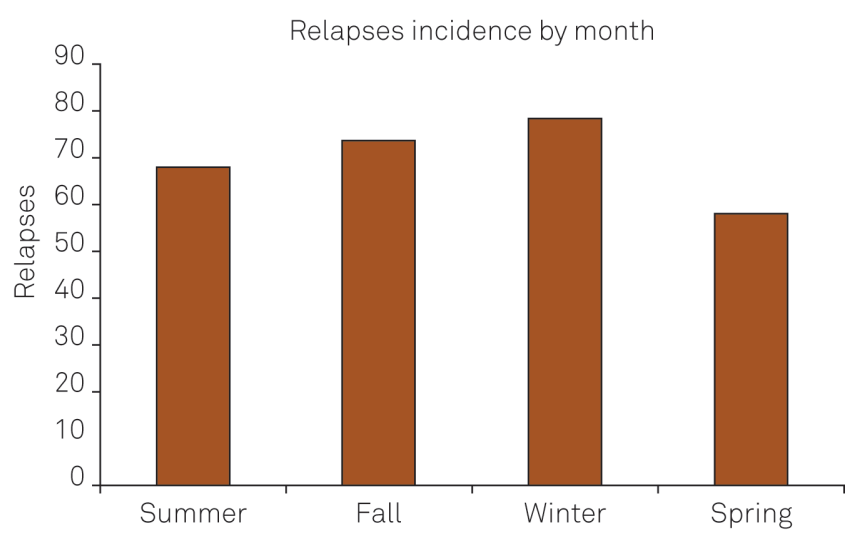

Figure: Relapse incidence according to month and season in 49 patients with NMO.

stress factors. Most of these patients received regular immunosuppressive treatment with azathioprine with or without prednisone as part of our regular treatment protocol, with $76 \%$ response $^{7}$, and thus treatment biases is expected to not have influenced these results.

In summary, we have evaluated 278 relapses from 49 patients with NMO and demonstrate no association between relapse incidence and seasonal variation, which is distinct to MS.

\section{Acknowledgements}

The authors would like to thank the Guthy Jackson foundation staff for bringing up the study idea.

Denis Bernardi Bichuetti, Manuela de Oliveira Fragomeni, Nilton Amorim de Souza, Enedina Maria Lobato Oliveira

\section{References}

1. Brum DG, Barreira AA, Santos AC, Kaimen-Maciel DR, Matiello M, Costa RM et al. HLA-DRB association in neuromyelitis optica is different from that observed in multiple sclerosis. Mult Scler. 2010;16(1):21-9. http://dx.doi.org/10.1177/1352458509350741

2. Handel AE, Giovannoni G, Ebers GC, Ramagopalan SV. Environmental factors and their timing in adult-onset multiple sclerosis. Nat Rev Neurol. 2010;6(3):156-66. http://dx.doi.org/10.1038/nrneurol.2010.1

3. Dobson R, Giovannoni G, Ramagopalan S. The month of birth effect in multiple sclerosis: systematic review, meta-analysis and effect of latitude. J Neurol Neurosurg Psychiatry. 2013;84(4):427-32. http://dx. doi.org/10.1136/jnnp-2012-303934

4. Damasceno A, Von Glehn F, Deus-Silva L, Damasceno BP. Monthly variation of multiple sclerosis activity in the southern hemisphere: analysis from 996 relapses in Brazil. Eur J Neurol. 2012;19(4):660-2. http://dx.doi.org/10.1111/j.1468-1331.2011.03543.x

5. Muto M, Mori M, Sato Y, Uzawa A, Masuda S, Kuwabara S. Seasonality of multiple sclerosis and neuromyelitis optica exacerbations in Japan. Mult Scler. 2013;19(3):378-9. http://dx.doi.org/ 10.1177/1352458512452332

6. Bichuetti D, Oliveira E, Souza N, Rivero R, Gabbai A. Neuromyelitis optica in Brazil: a study on clinical and prognostic factors. Mult Scler. 2009;15(5):613-9. http://dx.doi.org/10.1177/1352458508101935

7. Bichuetti DB, Oliveira EML, Oliveira DM, Souza NA, Gabbai AA. Neuromyelitis optica treatment: analysis of 36 patients. Arch Neurol. 2010;67(9):1131-6. http://dx.doi.org/10.1001/archneurol.2010.203 